\title{
Mögliche gesundheitliche Auswirkungen des Klimawandels in der Schweiz
}

\author{
O. Thommen ${ }^{a}$, L. Grize ${ }^{a}$, A. Huss ${ }^{a, b}$, C. Schindler ${ }^{a}$, C. Braun-Fahrländer ${ }^{a}$
}

\section{Einleitung}

Seit dem Hitzesommer 2003 sind auch in der Schweiz mögliche gesundheitliche Auswirkungen einer Klimaerwärmung vermehrt ins öffentliche Bewusstsein gerückt. Wir berichten hier über eine Untersuchung, die das Institut für Sozial- und Präventivmedizin der Universität Basel im Auftrag des Bundesamtes für Umwelt, Wald und Landschaft und des Bundesamtes für Gesundheit durchgeführt hat. Dabei wurden einerseits die Auswirkungen des Hitzesommers auf die Sterblichkeit in der Schweiz untersucht und andererseits mögliche indirekte Folgen der Klimaerwärmung für die Schweiz anhand der neueren wissenschaftlichen Literatur zusammengestellt.

\section{Klimaerwärmung in der Schweiz}

Die Wissenschaftler des Intergovernmental Panel of Climate Change (IPCC) hielten in ihrem Bericht fest, dass für das 20. Jahrhundert eine $\mathrm{Zu}$ nahme der globalen Jahresmitteltemperatur der Erdoberfläche um etwa $0,6^{\circ} \mathrm{C}$ festzustellen war, was dem höchsten Temperaturanstieg der letzten 1000 Jahre gleichkommt [1]. Der Grossteil der in den letzten 50 Jahren beobachteten Erwärmung ist menschlichen Aktivitäten zuzuschreiben [1]. In der Schweiz fand im 20. Jh. jedoch ein deutlich stärkerer Temperaturanstieg als im globalen Mittel $\left(0,6^{\circ} \mathrm{C}\right)$ statt und es wurden regionale Unterschiede zwischen der West- und Ostschweiz und zwischen der Alpennordseite und der Alpensüdseite beobachtet (Deutschschweiz $1,3^{\circ} \mathrm{C}[1,2-1,5]$, Westschweiz $1,6^{\circ} \mathrm{C}[1,5-1,7]$, Alpensüdseite $1,0^{\circ} \mathrm{C}$ ) [2]. Gemäss IPCC wird von einer Zunahme der Häufigkeit und/oder Intensität von sommerlichen thermischen Extremen in Mitteleuropa ausgegangen [1]. Es ist daher anzunehmen, dass es in Zukunft vermehrt Hitzesommer wie im Jahr 2003 geben wird.

Korrespondenz:

Oliver Thommen

Institut für Sozial- und Präventivmedizin der Universität Basel

Steinengraben 49

CH-4051 Basel

Tel. 0612702214

Fax 0612702225

E-Mail: oliver.thommen@unibas.ch
Auswirkungen der Hitzewelle auf die Sterblichkeit der Bevölkerung im Sommer 2003 haben vor allem in Frankreich für Schlagzeilen gesorgt.

\section{Direkte Hitzewirkung - Hitzewellen}

Zwischen dem 1. und 20. August wurden in Frankreich 14800 zusätzliche Todesfälle registriert, in Paris betrug die Übersterblichkeit 138\% [3]. Wie die Analysen des Hitzesommers in der Schweiz zeigten, sind in den drei Sommermonaten 2003 über 900 Personen (7\%) mehr gestorben als statistisch zu erwarten gewesen wären [4]. Eine erhöhte Sterblichkeit zeigte sich insbesondere in den Städten und Agglomerationen der Alpennordseite (Basel [+24\%], Genf [+17,5\%], Lausanne $[+13,5 \%]$ ) sowie bei älteren Personen. Die Kombination von hohen Temperaturen am Tag und ausbleibender Abkühlung nachts trat vor allem in den Städten Basel und Genf auf, wo die stärkste Zunahme der Sterblichkeit festgestellt wurde. Die erhöhte Mortalität während des Hitzesommers war nicht alleine durch den vorzeitigen Tod von bereits schwerkranken Personen erklärbar, denn die Gesamtzahl der Todesfälle blieb bis im Dezember höher als in den Vorjahren. Auch Studien aus Deutschland, Italien, Spanien, England und den Niederlanden dokumentieren eine Zunahme der Sterblichkeit während der Hitzewelle 2003 [4]. Gesundheitliche Auswirkungen von Hitzewellen sind in zahlreichen Studien, insbesondere aus den USA, belegt. Insgesamt zeigen diese Untersuchungen, dass die Mehrzahl der Todesfälle während Hitzewellen auf Herzkreislauf-, Hirngefäss- und Atemwegserkrankungen zurückzuführen ist [5]. Zu den Risikogruppen gehören ältere Personen und Kleinkinder, bereits geschwächte bzw. kranke Personen und Menschen mit niedrigem sozioökonomischem Status. Daneben sozial isolierte und bettlägerige Menschen sowie psychisch Kranke [6, 7]. Prädisponierende Faktoren für hitzebedingte Krankheiten und/oder hitzebedingten Tod sind zudem mangelnde Fitness, Übergewicht, Müdigkeit, Schlafmangel, Dehydrierung aufgrund zu geringer Nahrungsmittelund Flüssigkeitsaufnahme, Alkoholmissbrauch und Einnahme gewisser Medikamente (z.B. Diuretika) [7]. Wie Erfahrungen aus Nordamerika zeigen, kann die (hitzebedingte) Mortalität durch rechtzeitige Warnungen vor Hitzewellen und Informationen zu adäquatem Verhalten wirkungsvoll gesenkt werden $[7,8]$. 


\section{Ozon}

Langandauernde Schönwetterperioden und hohe Temperaturen begünstigen auch die Bildung von Ozon aus Stickoxiden $\left(\mathrm{NO}_{\mathrm{x}}\right)$ und flüchtigen organischen Verbindungen (VOC). Während des Sommers 2003 wurde in der Schweiz der Ozongrenzwert von $120 \mu \mathrm{g} / \mathrm{m}^{3}$ an den NABEL-Messstationen rund doppelt so häufig überschritten wie in den vergangenen Jahren [9]. Erhöhte Ozonkonzentrationen sind in zahlreichen Studien mit einer Zunahme der Sterblichkeit assoziiert worden. Die Europäische APHEA-Studie (Air Pollution and Health, a European Approach), in der auch Sterblichkeitsdaten aus den Städten Basel, Zürich und Genf berücksichtigt wurden, kam zum Schluss, dass die Sterbefälle in den Sommermonaten um 0,3\% zunahmen, wenn die 8-Stunden-Mittelwerte von Ozon um $10 \mu \mathrm{g} / \mathrm{m}^{3}$ anstiegen. Die kardiovaskuläre Mortalität stieg um $0,45 \%$, die respiratorische Mortalität um 1,1\% an. Diese Wirkungen konnten dem Ozon zugeordnet werden und waren unabhängig von den Wirkungen der Temperatur und der Feinstaub- und Stickstoffdioxidbelastung [10].

Während einer Hitzewelle sind die Wirkungen von Temperatur und Ozon oft schwierig zu trennen, da beide Parameter gleichzeitig ansteigen. In längeren Zeitreihenuntersuchungen und mit entsprechenden statistischen Methoden können die Effekte aber getrennt berechnet werden [11]. Aufgrund der erwähnten DosisWirkungs-Beziehung zwischen Ozon und Sterblichkeit sowie der Ozonexposition der Schweizer Bevölkerung im Sommer 2003 konnte berechnet werden, dass etwa 130-300 (13-30\%) der beobachteten vorzeitigen Todesfälle auf die übermässige Ozonbelastung zurückzuführen sind [9].

\section{Indirekte Wirkungen der Klima- erwärmung}

\section{Pollenflug und allergische Erkrankungen}

In den vergangenen Jahren hat eine Reihe von Studien den Einfluss von klimatischen Veränderungen auf das Vorkommen von luftgetragenen Allergenen (Pollen und Pilzsporen) untersucht [12].

In experimentellen Untersuchungen konnte z. B. gezeigt werden, dass eine Verdoppelung der $\mathrm{CO}_{2}$-Konzentration zu einer erhöhten Produktion von Weidelgraspollen (ryegrass) führte [13].

In der Schweiz untersuchte Frei [14] den zeitlichen Verlauf der Konzentrationen von Hasel-, Birken- und Graspollen von 1969 bis 1996 und den gleichzeitigen Einfluss von Klimafaktoren. In diesem Zeitraum zeigt die Pollenkonzentra- tion einen ansteigenden Trend, der parallel mit dem Anstieg der Temperatur verlief. Auch konnte nachgewiesen werden, dass die Pollensaison im Frühjahr früher beginnt und sich dadurch die Pollensaison verlängert. Ähnliche Ergebnisse wurden aus andern Untersuchungen in Europa (England, Dänemark, Italien), Nordamerika und Japan berichtet. Wenn mehr Pollen produziert und mit dem Wind verbreitet werden, besteht auch die Möglichkeit, dass die Zahl der pollenproduzierenden Pflanzen zunimmt. Ob die Zunahme der Pollenkonzentration mit einer stärkeren allergenen Wirkung einhergeht, ist allerdings nicht gesichert.

Allergene von Pollen und Pilzsporen spielen eine wichtige Rolle bei der Auslösung von Symptomen bei Personen, die an Asthma oder Heuschnupfen leiden. Mehrere Untersuchungen zeigten, dass die Zahl der Patienten, die wegen eines akuten Asthmaanfalls hospitalisiert werden mussten, bei erhöhten Konzentrationen von Pilzsporen oder Pollen ansteigt [15]. Asthmaepidemien liessen sich gemäss einer englischen Studie jedoch nur in 15\% der Fälle aufgrund der Pollenkonzentrationen vorhersagen. Experimentelle und einzelne epidemiologische Studien kommen zum Schluss, dass die Wirkung von Pollen durch erhöhte Konzentrationen von Luftschadstoffen, insbesondere des Ozons, verstärkt wird. Eine Verlängerung der Pollensaison bedeutet für betroffene Patienten auch eine längere Periode, während der gesundheitliche Beschwerden auftreten können.

Faktoren, die das Auslösen von Beschwerden bei bereits erkrankten Personen bewirken, sind aber nicht notwendigerweise verantwortlich für die Entstehung dieser Krankheiten. So wird heute zunehmend in Frage gestellt, ob erhöhte Allergenkonzentrationen tatsächlich an der Entstehung von Asthma und Allergien beteiligt sind. So ist in der Schweiz und einigen andern Ländern (Deutschland, Italien, England, Australien) seit Beginn der 1990er Jahre eine Stabilisierung der Asthma- und Heuschnupfenraten beobachtet worden [16], obwohl im gleichen Zeitraum die Pollenkonzentrationen eher zugenommen haben.

\section{Vektorübertragene Krankheiten}

Wärmere Temperaturen können zu Veränderungen von Ökosystemen führen und sich dadurch indirekt auf die menschliche Gesundheit auswirken. Viele Vektororganismen regulieren ihre Körpertemperatur nicht selbst. Somit sind sie in ihrer Verbreitung und Reproduktion stark von der Temperatur, der Feuchtigkeit und weiteren klimabedingten Umweltfaktoren wie Wind, 
Oberflächenwasser, Bodenfeuchte und Waldverbreitung abhängig [17]. Erhöhte Temperaturen und Feuchtigkeit verbessern die Lebensumstände der meisten Parasiten und können somit das saisonale und regionale Auftreten von vektorübertragenen Krankheiten begünstigen. Malaria und Dengue-Fieber sind die wichtigsten vektorbedingten Krankheiten in den Tropen und Subtropen. In der Schweiz blieben die gemeldeten Malariafälle in den letzten zehn Jahren stabil und betrugen für die Jahre 2001 und 2002 durchschnittlich 3,7 Fälle pro 100000 Einwohner. Bei Schweizer Patienten ist die Mehrzahl der Fälle auf den Tourismus zurückzuführen, wogegen bei Einwohnern ausländischer Herkunft Besuche bei der Familie im Herkunftsland häufigste Ursache für eine Malariaerkrankung sind [18]. Bei einem Temperaturanstieg von 3 bis $5^{\circ} \mathrm{C}$ bis zum Jahr 2100 könnte sich gemäss Hochrechnungen die Übertragungsgefahr von Malaria in tropischen Regionen verdoppeln, in gemässigten Gebieten sogar mehr als verzehnfachen. Auch in Mitteleuropa müsste unter solchen Bedingungen mit einer künftigen Ausbreitung von Malaria gerechnet werden [1].

In den USA und Europa verbreitet ist die Lyme-Borreliose, die häufigste vektorübertragene Krankheit, und in zunehmendem Masse auch die Zeckenenzephalitis.

Empirische Untersuchungen in Schweden zeigten erstmals einen plausiblen Zusammenhang zwischen der Umgebungstemperatur und der Verbreitung von Zeckenenzephalitis. Das relative milde Klima der 1990er Jahre in Schweden hatte zur Folge, dass sich die Zecken (Ixodes ricinus) weiter nach Norden ausbreiten konnten. Die milden Winter der 1990er Jahre und die längeren Sommer- und Frühjahrsperioden bewirkten eine Zunahme der Zeckendichte. In Schweden hat sich seit Mitte der 1980er Jahre die Inzidenz der Zeckenenzephalitis deutlich erhöht $[19,20]$. Ob es sich hier jedoch um kausale $\mathrm{Zu}$ sammenhänge handelt, kann aufgrund dieser Untersuchungen nicht mit Sicherheit gesagt werden. Eine erhöhte Exposition gegenüber Zecken kann auch durch Veränderungen im Freizeitverhalten der Bevölkerung zustande kommen. Eine stärkere Vermehrung der Parasiten kann zudem durch Änderungen der landwirtschaftlichen Nutzung (Anlegen von Feuchtbiotopen, Brachen) begünstigt werden.

In der Schweiz sind 5-30\% der Zecken mit dem Bakterium Borrelia burgdorferi infiziert. Etwa 3000 Personen erkranken jedes Jahr an LymeBorreliose, ungefähr 95 Personen an Zeckenenzephalitis. Zecken, welche das Virus be- herbergen, sind auf einzelne Endemiegebiete (Naturherde) beschränkt, die sich in den Kantonen Thurgau, Aargau, Zug, St. Gallen, Luzern, Bern, Graubünden, Schaffhausen, Solothurn und Zürich befinden (www.bag.admin.ch/infekt/ krank/d/encephalite.htm).

Anhand von Satellitendaten und Klimamodellen wird prognostiziert, dass sich die Verbreitung der Zeckenenzephalitis im 21. Jahrhundert sukzessive in höhere Breitengrade und Höhenlagen verschieben könnte. Damit könnte die Schweiz bis 2020 frei vom Zeckenenzephalitisvirus sein [21].

Aus klimatischen Gründen spielen vektorübertragene Krankheiten bis anhin in der Schweiz eine untergeordnete Rolle. In Anbetracht der prognostizierten Klimaerwärmung kommt der Überwachung von Vektoren und den von ihnen übertragenen Krankheiten als Voraussetzung für eine adäquate Prophylaxe und Bekämpfung jedoch wachsende Bedeutung zu. Das Bundesamt für Veterinärwesen startet $a b$ Frühjahr 2005 ein Frühwarnsystem für vektorübertragene Krankheiten bei Nutztieren in der Schweiz (www.bvet.admin.ch/bvet/00302/00305/00561/ index.html?lang=de). Da Krankheiten, die in einem Sektor (Tiermedizin) auftreten, möglicherweise Hinweise auf zukünftige Entwicklungen im Humanbereich geben [22], wäre ein sektorübergreifender Austausch von Behörden und Wissenschaft dringend erforderlich.

\section{Nahrungsmittelübertragene Infektions- krankheiten}

Campylobacter und Salmonella sind die von den mikrobiologisch-diagnostischen Labors am häufigsten nachgewiesenen bakteriellen Erreger bei Durchfallerkrankungen in der Schweiz (Campylobacter 200378 Fälle pro 100000 Einwohner und Salmonella 200331 Fälle pro 100000 Einwohner). Beide Erreger zeigen typische saisonale Muster mit sommerlichen Maxima. Seit 1999 ist für beide Erreger die Zahl der beim Bundesamt für Gesundheit gemeldeten Fälle rückläufig [23].

\section{Campylobacter}

Eine Übertragung von Campylobacter kann über das Wasser, Nahrungsmittel, insbesondere kontaminiertes Geflügelfleisch, Milch oder direkt aus der Umwelt erfolgen. Campylobacter vermehrt sich jedoch nicht in Lebensmitteln. Eine kürzlich veröffentlichte Studie untersuchte erstmals die Rolle der Umgebungstemperatur auf das saisonale Auftreten von Campylobacter-Infektionen in 15 europäischen Ländern, darunter auch der Schweiz [24]. Die Untersuchung ergab 
jedoch keinen wesentlichen Einfluss der Temperatur auf das saisonale Muster von Campylobacter-Infektionen. Die Gründe für die sommerliche Infektionshäufung bleiben somit ungeklärt. Dies schliesst jedoch nicht aus, dass einzelne Ausbrüche mit den besonderen klimatischen Bedingungen des Hitzesommers 2003 in Zusammenhang standen. So berichtete das BAG von einem Campylobacter-Ausbruch, als dessen Ursache das Verwenden von ungekochtem Wasser aus einem Bach, der wenig Wasser führte, vermutet wird [23].

\section{Salmonella}

Der nach wie vor häufigste Serotyp ist $S$. Enteritidis (80,1\% im Jahr 1992, 53,4\% im Jahr 2003). S. Typhimurium war im selben Zeitraum, mit leicht zunehmendem Anteil, immer an zweiter Stelle (2003: 22\%). Rohe tierische Lebensmittel (Eier, Fleisch) stellen das grösste Infektionsrisiko dar [23].

Der Zusammenhang zwischen Umgebungstemperatur und Auftreten von Salmonelleninfektionen wurde in mehreren Studien untersucht [25], u.a. auch aufgrund von Meldedaten aus der Schweiz [26].

Übereinstimmend belegen diese Untersuchungen einen klaren Zusammenhang zwischen der Häufigkeit von Salmonelleninfektionen und den Temperaturen, die in den Wochen vor den Krankheitsausbrüchen gemessen wurden. Gemäss der Zeitreihenanalyse mit Daten von zehn europäischen Ländern besteht zwischen den gemeldeten Salmonelleninfektionen und der jeweiligen Umgebungstemperatur über einem Schwellenwert von $6{ }^{\circ} \mathrm{C}$ eine lineare Beziehung [26]. Infektionen mit $S$. Enteritidis wiesen einen stärkeren Zusammenhang mit der Umgebungstemperatur auf als Infektionen mit S. Typhimurium. Etwa 35\% der Ansteckungen können auf den Effekt der Temperatur zurückgeführt werden.

Es ist davon auszugehen, dass es sich dabei nicht ausschliesslich um einen direkten Einfluss der Temperatur auf die Vermehrungsraten der Salmonellen handelt. Warmes Sommerwetter verändert auch die Essgewohnheiten. So sind beim Grillieren im Freien und beim Konsum roher oder leicht erhitzter Speisen die für eine Inaktivierung von Salmonellen notwendigen Bedingungen nicht immer gegeben.

Obwohl die Anzahl der Lebensmittelinfektionen dank aktiver Kontrollmassnahmen rückläufig ist, sind somit in bezug auf den Umgang mit Lebensmitteln im Hochsommer (Lagerung, Zubereitung, Konsum) besondere Informationsbzw. Präventionsanstrengungen von seiten der Gesundheitsbehörden notwendig.

\section{Schlussfolgerungen}

Für die Schweiz sind die direkten und indirekten gesundheitlichen Auswirkungen von hohen sommerlichen Temperaturen die wahrscheinlichsten Folgen der Klimaerwärmung. Wie sich eine Zunahme milderer Winter auf die Gesundheit auswirken wird, ist derzeit schwierig abzuschätzen, da hier Influenzaepidemien, die nicht in einem direkten Zusammenhang zur Temperatur stehen, eine wichtige Rolle spielen.

Mit gezielten Informationen und einer rechtzeitigen Warnung vor extremen Wetter- und Klimabedingungen lassen sich die negativen Auswirkungen auf die Gesundheit wirkungsvoll beeinflussen. Dies ist einerseits Aufgabe der Gesundheitsbehörden von Bund und Kantonen in Zusammenarbeit mit den meteorologischen Diensten. Einzelne Kantone (Tessin, Genf) haben hier schon wichtige Vorarbeit geleistet. Andererseits kommt auch den Hausärztinnen/-ärzten eine wichtige Rolle bei der Beratung und Information ihrer Patienten bezüglich Verhaltensanpassungen bei Hitze zu.

Bei den Recherchen für diese Untersuchungen ist uns immer wieder aufgefallen, dass in der Schweiz zwar Daten zur Gesundheit in verschiedenen Meldesystemen erfasst werden, diese aber kaum unter dem Aspekt der Klimasensitivität betrachtet werden.

Für das zukünftige Monitoring von möglichen Auswirkungen der Klimaerwärmung auf die Gesundheit sollte die Mortalitätsstatistik regelmässig im Zusammenhang mit der Temperatur analysiert werden. Für die Untersuchung einer Hitzeepisode ist ein schnellerer Zugriff auf die Daten, insbesondere die Todesursachenstatistik, dringend erforderlich. Zum andern sollte der sektorübergreifende Austausch von Informationen bezüglich Vektoren und Nahrungsmittelkeimen unter dem Aspekt des Klimas in Form einer Arbeitsgruppe institutionalisiert werden. Diese werden sich mit der Überwachung von klimasensitiven Infektionen beschäftigen, wobei Personen aus dem Bereich der Human- und der Veterinärmedizin, aus Verwaltung und Wissenschaft darin vertreten sein müssten. 


\section{Literatur}

1 IPCC. Climate Change 2001: Impacts, Adaptation and Vulnerability. Contribution of Working Group II to the Third Assessment Report of the Intergovernmental Panel on Climate Change. Cambridge: Cambridge University Press; 2001.

2 OcCC. Das Klima ändert - auch in der Schweiz. Die wichtigsten Ergebnisse des dritten Wissensstandsberichts des IPCC aus der Sicht der Schweiz. Bern: OcCC; 2002.

3 Vandentorren S, Suzan F, Medina S, Pascal M, Maulpoix A, Cohen JC, et al. Mortality in 13 French cities during the August 2003 heat wave. Am J Public Health 2004;94(9):1518-20.

4 Grize L, Huss A, Thommen O, Schindler C, BraunFahrländer C. Heat wave 2003 and mortality in Switzerland. Swiss Med Wkly 2005;135:200-5.

5 Braga AL, Zanobetti A, Schwartz J. The effect of weather on respiratory and cardiovascular deaths in 12 U.S. cities. Environ Health Perspect 2002; 110(9):859-63.

6 Bark N. Deaths of psychiatric patients during heat waves. Psychiatr Serv 1998;49(8):1088-90.

7 WHO. Heat-waves: risks and responses. Copenhagen: WHO; 2004.

8 Smoyer KE. A comparative analysis of heat waves and associated mortality in St. Louis, Missouri 1980 and 1995. Int J Biometeorol 1998;42(1): 44-50.

9 EKL. Sommersmog. Bern: Eidgenössische Kommission für Lufthygiene; Juni 2004.

10 Gryparis A, Forsberg B, Katsouyanni K, Analitis A, Touloumi G, Schwartz J, et al. Acute effects of ozone on mortality from the «air pollution and health: a European approach» project. Am J Respir Crit Care Med 2004;170(10):1080-7.

11 Schwartz J. How sensitive is the association between ozone and daily deaths to control for temperature? Am J Respir Crit Care Med 2005; 171(6):627-31.

12 Beggs PJ. Impacts of climate change on aeroallergens: past and future. Clin Exp Allergy 2004 34(10):1507-13.

13 Wayne P, Foster S, Connolly J, Bazzaz F, Epstein P. Production of allergenic pollen by ragweed (Ambrosia artemisiifolia L.) is increased in $\mathrm{CO}_{2-}$ enriched atmospheres. Ann Allergy Asthma Immunol 2002;88(3):279-82.
14 Frei T. The effects of climate change in Switzerland 1969-1996 on airborne pollen quantities from hazel, birch and grass. Grana 1998;37:172-9.

15 Dales RE, Cakmak S, Judek S, Dann T, Coates F, Brook JR, et al. Influence of outdoor aeroallergens on hospitalization for asthma in Canada. J Allergy Clin Immunol 2004;113(2):303-6.

16 Braun-Fahrlander C, Gassner M, Grize L, Takken-Sahli K, Neu U, Stricker T, et al. No further increase in asthma, hay fever and atopic sensitisation in adolescents living in Switzerland. Eur Respir J 2004;23(3):407-13.

17 McMichael AJ. Global climate change: will it affect vector-borne infectious diseases? Intern Med J 2003;33(12):554-5

18 BAG. Malaria in der Schweiz: In den Jahren 2001 und 2002 erfasste Fälle. BAG-Bulletin 2004;4:44-9.

19 Lindgren E, Gustafson R. Tick-borne encephalitis in Sweden and climate change. Lancet 2001; 358(9275):16-8.

20 Lindgren E, Talleklint L, Polfeldt T. Impact of climatic change on the northern latitude limit and population density of the disease-transmitting European tick Ixodes ricinus. Environ Health Perspect 2000;108(2):119-23.

21 Randolph S. Predicting the risk of tick-borne diseases. Int J Med Microbiol 2002;291(Suppl 33) 6-10.

22 Zinsstag J, Schelling E. Vector-borne diseases in humans and animals: activities of the Swiss Tropical Institute and risks for Switzerland. Schweiz Arch Tierheilkd 2003;145(12):559-66, 568-9

23 BAG. Campylobacter und Salmonella - Stand Ende 2003. Epidemiologie und Infektionskrankheiten BAG-Bulletin 2004;40:737-40.

24 Sari Kovats R, Edwards SJ, Charron D, Cowden J, D'Souza RM, Ebi KL, et al. Climate variability and campylobacter infection: an international study. Int J Biometeorol 2005;49(4):207-14.

25 D'Souza RM, Becker NG, Hall G, Moodie KB. Does ambient temperature affect foodborne disease? Epidemiology 2004;15(1):86-92.

26 Kovats RS, Edwards SJ, Hajat S, Armstrong BG, Ebi KL, Menne B. The effect of temperature on food poisoning: a time-series analysis of salmonellosis in ten European countries. Epidemiol Infect 2004;132(3):443-53. 